\title{
Denoising Algorithm of Airborne LIDAR Point Cloud Based on 3D Grid
}

\author{
Shan Yong-hua ${ }^{1}$, Zhang Xu-qing ${ }^{1}$, Niu Xue-feng ${ }^{1}$, Yang guo-dong ${ }^{1}$ and Zhang \\ $\mathrm{Ji}-\mathrm{Kai}^{1}$ \\ (School of Earth Exploration Science and Technology Jilin University Changchun \\ 130026 China $)^{1}$ \\ E-mail:(1311712003@qq.com,zxq@jlu.edu.cn,nxf@jlu.edu.cn,ygd@jlu.edu.cn, \\ 18641692195@qq.com)
}

\begin{abstract}
The first step of the airborne laser lidar point cloud processing is the removal of noise points in the point cloud data, which has a great influence on the following point cloud filtering processing. Through the 3D space grid point, the cloud data of the inside of each cubic grid points have the property of spatial index, according to the spatial neighborhood relationship between $3 D$ grid judgments within the grid point as noise points. Experimental results show that this algorithm can effectively filter the terrain point cloud of the discrete noise points and clusters of small noise, by using appropriate correlation coefficient of the average distance between the $3 D$ grid sidescan which greatly reduces the error of determining noise.
\end{abstract}

Keywords: airborne LIDAR; point cloud denoising; $3 D$ grid; neighborhood analysis; average point distance

\section{Introduction}

With the rapid development of the 3D laser scanning technology, people can easily obtain objects in the real world from the 3D surface point cloud data, so the 3D point cloud data model is widely used in virtual reality, reverse engineering, urban modeling etc[1].Airborne LIDAR in the scanning process when obtaining point cloud data with a large number of non-surface points acquired, which may be atmospheric impurities, flying bird or maximum below the surface and error almost will, in general, these points is referred to as noise points. These noise points must be filtered before the filtering process of point cloud data, the presence of noise points will make the original point cloud data to generate Digital Terrain Model (DTM) result in great error, at the same time in the use of most point cloud filtering process, the noise points which greatly lower the surface will resulting in serious filtering error [2]. Also the existence of noise point will make great impact in subsequent large-scale point cloud hierarchical rendering, object recognition extraction, model building and dense matching income of the same name point cloud of the subsequent processing precision (as a shadow image matching, object recognition, etc.)[3-4], which makes the airborne LIDAR acquisition point cloud denoising processing become more and more important.

The 3D surface point cloud data noise gross error is mainly reflected in elevation values, its remarkable characteristic is than around the point of a large relative elevation, showing an isolated singular values. There are mainly two kinds, namely high outlier and low outlier. From the form of existence, Gross errors difference to outlier clusters and isolated outliers. Outlier clusters clustered in groups, isolated outliers exist in the form of isolated point [2].

Many scholars have made deep research on the point cloud denoising: Spiros (2002) classified and summarized the existing methods of gross error elimination. It can be 
divided into five types, which is based on the distribution, depth, clustering, distance and density of gross error elimination method [5]. Gross error elimination method based on distribution, such as Nie Jianhui[6] proposed the classification and recognition algorithm of scattered points, according to the surface variation based on the local outlier factor(SVLOF) method to classify the outliers, removal, Cao Shuang[7] proposed based on feature selection of bilateral filtering point clouds to denoising algorithm, to classify the noise, feature and non-feature points of bilateral filtering factor calculation and avoid bilateral filtering and excessive smoothing phenomenon, but requires a longer period of time to determine the feature selection. These algorithms are very effective for statistical rule of data, but due to the point cloud data model is difficult to estimate and the application of SVLOF have certain limitations. Based on depth to eliminate gross errors, it is necessary to calculate the data set of geometry and dimension of the convex hull of different layers and located in the outermost point cloud that is gross error. This algorithm by data dimension constraints. Gross error elimination based on clustering, Zhang Qiaoying[8] proposed the continuous distribution of point cloud based on density clustering algorithm to denoising algorithm, is to divide the data into a number of clusters, are excluded in the clustering of the data that is noise. Based on density detection of outliers, Zhu Junfeng[9] proposed multiscale point cloud noise detection density analysis method to distinguish noise using the local density, the algorithm need to specify a range of minimum number and density, the method set a point as the center calculated the neighborhood range laser feet point density, if the density less than the given threshold that the point is gross error. The noise eliminated methods above have their own advantages and disadvantages, every method can eliminate the portion of gross error basically, occasionally because of some special noise point, judging may fail. Point cloud noise can be considered as the elevation deviation within the scope of the "neighborhood" point "far" and therefore the point cloud noise detection of the key lies in the neighborhood and to determine the threshold selection. This paper through the MATLAB platform using $\mathrm{C}$ language programming environment for the establishment of point cloud data of the 3D space grid, with an average spacing grid threshold reference coefficient, through the spatial neighborhood relation between each grid cell, the discrete point cloud body of isolated and clustered terrain point cloud noise points respectively the spatial index and diffusion operation way to filter out noise.

\section{Denoising Algorithm based on Three-Dimensional Grid}

\subsection{The 3d Grid Point Cloud}

The basic idea of point cloud 3D grid with a grid spacing $\mathrm{D}$ respectively on the point cloud data connection cuboid XYZ directions are used to segment and hexahedral space as the basic unit established point cloud of 3D grid index. The 3D grid index to establish specific process is as follows : According to the point cloud point XYZ coordinates of the extreme value to determine the point cloud external rectangle, that is $\mathrm{X} \in[\mathrm{XMIN}, \mathrm{XMAX}] \mathrm{Y} \in[\mathrm{YMIN}, \mathrm{YMAX}] 、 \mathrm{Z} \in[\mathrm{ZMIN}, \mathrm{ZMAX}]$.Establish attributes table M1 aim at point cloud XYZ coordinates and the position of each point belongs to 3D grid, which contains the fields that are XYZ coordinate value, the 3D grid spatial V1 index value, the 3D grid spatial V2 index value.

The coordinates of point cloud data points are stored in column 1th, 2th, 3th of the table. The circumscribed cuboid is placed along the XYZ axis respectively by setting $\mathrm{uD}$ sequence segmentation, where $u$ is the grid spacing coefficient. The Schematic diagram of segmentation is shown in Figure 1. 


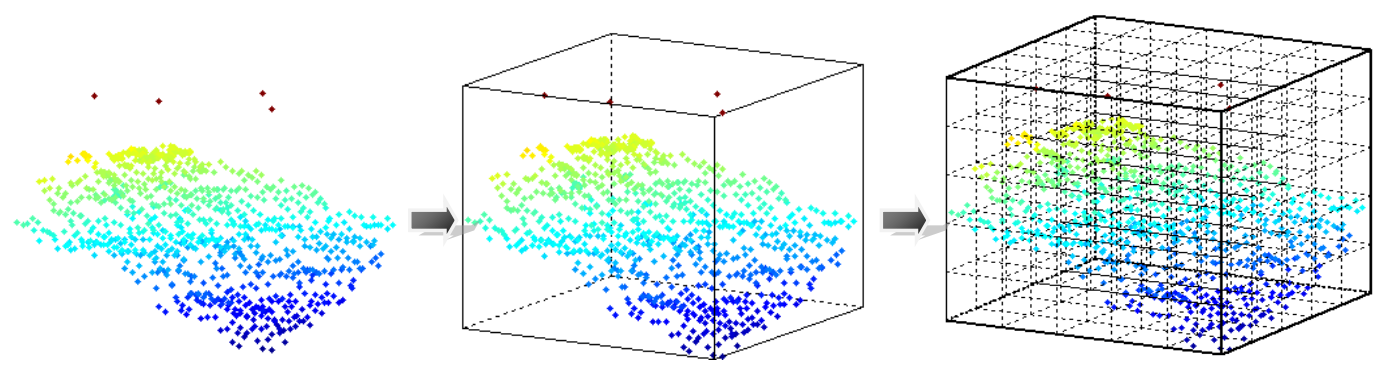

a. Original point cloud

b. External rectangle

C. Point cloud $3 \mathrm{D}$ grid $\mathrm{V}_{1}$

\section{Figure 1. Schematic Diagram of 3D Grid Segmentation}

Establishing spatial index for each Cubic lattice in 3D grid V1: $\mathrm{N}_{\mathrm{Xmax}} * \mathrm{~N}_{\mathrm{Ymax}} * \mathrm{~N}_{\mathrm{Zmax}}$, among them N_Xmax $=\operatorname{int}\left\{\left(X_{-}\right.\right.$MAX $-X_{-}$MIN $\left.) / u D\right\}+1$, N_Ymax $=\operatorname{int}\left\{\left(Y \_M A X-\right.\right.$ Y_MIN $) / u D\}+1, \quad$ N_Zmax $=\operatorname{int}\left\{\left(Z_{-} \_M A X-Z_{-}\right.\right.$MIN $\left.) / u D\right\}+1$.The index value is numbered in the form of three-dimensional coordinates, Such as the first row of first rows of first columns of the cubic grid number $(1,1,1)$, the third row of the fifth row of the seventh column of the cubic grid number $(3,5,7) \ldots \ldots$. Taking the point data in point cloud associate with the corresponding cubic lattice in 3D grid. According to the range of grid segmentation of each grid in coordinate space coordinates of the XYZ, Matches the point in the corresponding range with the cube grid. The index values of each point in the three dimensional grid are stored in the $4^{\text {th }}, 5^{\text {th }}, 6^{\text {th }}$ column of the XYZ table, remember to InJn $\mathrm{Kn}$ (Where $\mathrm{n}$ is the number of the two-dimensional tables in $\mathrm{M}$ ), So that every point $\mathrm{M}$ (n) in the point cloud can be indexed to the corresponding cubic lattice $(\mathrm{In}, \mathrm{Jn}, \mathrm{Kn})$ in the outer cube.

Establishing a new three-dimensional grid index $\mathrm{V} 2:\left(\mathrm{N}_{\mathrm{X} \max }+2\right) *\left(\mathrm{~N}_{\mathrm{Ymax}}+2\right) *$ $\left(\mathrm{N}_{\mathrm{Zmax}}+2\right)$, the purpose is expand the boundary of $3 \mathrm{D}$ grid peripheral cuboid the existing cut into the grid, therefore, the window structure consists of 27 cubic grid index out of bounds will not appear in the process of 3D grid traversal, Reduced the complexity of the traversal algorithm. The specific effects of grid index as shown in Figure 2.

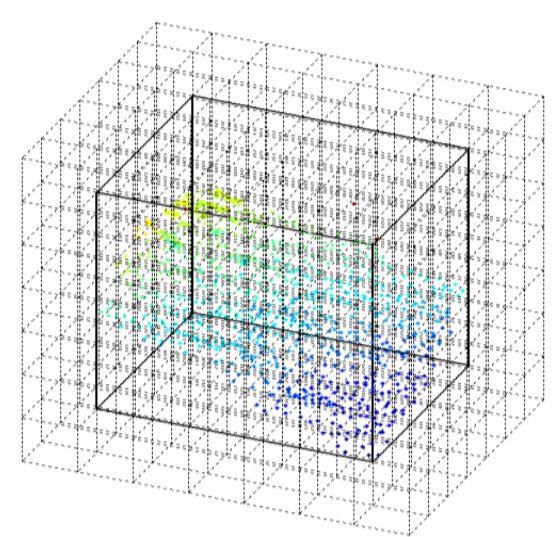

$\begin{array}{rrrrrrrrrr}0 & 0 & 0 & 0 & 0 & 0 & 0 & 0 & 0 & 0 \\ 0 & 0 & 0 & 0 & 0 & 0 & 0 & 0 & 0 & 0 \\ 0 & 0 & 0 & 0 & 0 & 0 & 0 & 0 & 0 & 0 \\ 0 & 0 & 0 & 0 & 0 & 0 & 0 & 0 & 0 & 0 \\ 6 & 45 & 5 & 0 & 0 & 0 & 0 & 0 & 0 & 0 \\ 7 & 47 & 22 & 1 & 0 & 2 & 1 & 0 & 0 & 0 \\ 2 & 32 & 39 & 23 & 1 & 17 & 1 & 1 & 1 & 3 \\ 1 & 6 & 2 & 4 & 39 & 10 & 72 & 72 & 44 & 2 \\ 1 & 9 & 4 & 0 & 12 & 8 & 2 & 9 & 20 & 0 \\ 4 & 0 & 10 & 17 & 2 & 0 & 1 & 11 & 12 & 15 \\ 0 & 0 & 0 & 2 & 0 & 0 & 0 & 8 & 31 & 11 \\ 2 & 1 & 1 & 0 & 0 & 0 & 0 & 2 & 14 & 38 \\ 0 & 2 & 1 & 10 & 1 & 0 & 1 & 14 & 6 & 9 \\ 0 & 3 & 1 & 6 & 2 & 0 & 2 & 1 & 1 & 7 \\ 0 & 0 & 1 & 2 & 0 & 0 & 0 & 0 & 1 & 23 \\ 0 & 2 & 0 & 0 & 0 & 0 & 7 & 5 & 6 & 4 \\ 0 & 0 & 0 & 2 & 0 & 0 & 0 & 1 & 18 & 5 \\ 0 & 0 & 4 & 4 & 2 & 0 & 1 & 1 & 7 & 0\end{array}$

a.index of 3D grid V2

b.2D diagram of the number of points in a cube lattice

\section{Figure 2. Schematic Diagram of Structure Window and Point Cloud} Segmentation

Matching the point cloud data to the new 3D grid index, And store the index value in the 7,8,9 column of table M1(which is $\mathrm{M} 1(\mathrm{n}, 7)=\mathrm{M} 1(\mathrm{n}, 4)+1, \mathrm{M} 1(\mathrm{n}, 8)=\mathrm{M} 1(\mathrm{n}, 5)+1, \mathrm{M} 1(\mathrm{n}, 9)$

$=\mathrm{M} 1(\mathrm{n}, 6)+1)$. On the basis of the two-dimensional table 7th 8th 9th column X Y Z cubic grid spatial index parameters, establishing corresponding cubic raster attribute list 
M2 to complete the point cloud data convert to 3D grid, The fields contained in M2are: Cubic raster space in 3D grid V2 position index. Each cube contains points inside the grid、Diffusion operator reserved field, The cubic grid space in 3D grid V2 index value is stored in the 1th 2 th 3 th column of table M2 (M2(:,1), M2(:,2), M2(:,3)), The number of points contained in each cube is stored in the 4th column. (M2(:,4)). The neighborhood relationship between the cube lattice and the number of points shown in Figure 3 . Building 3D grid brings the point cloud data which included in different cubic are endowed with different cubic grid index value, to make the spatial correspondence between the points that contain the points in different cubic grids, $\mathrm{n}$ order to carry out the spatial neighborhood topological analysis.

\subsection{Discrimination of Discrete Noise Points}

As the isolated outliers are the isolated data point which deviates from the point cloud, it is only needed to determine that the main body of the point cloud and other noise points are not included. The index structure window is composed of the center indexed cube which is surrounded by 26 cube grids, traverse the cube grid attribute table M2 and Extract M2 $(i, 4)=1$ corresponding to the array unit And to search for the cube grid cell neighborhood of 26 cube grid values are 0 or not, if all the cube grid cell neighborhood of 26 cube grid values are 0 , The point of the cubic lattice unit is judged as isolated outliers. as the algorithm is carried out only one cycle operation when judge the noise point grid, it is faster than the algorithm as the center point of the high grid index are established. Discrete noise 3D grid index as shown in Figure 3.

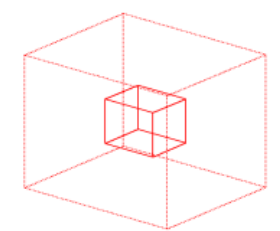

a.Schematic diagram of structure window

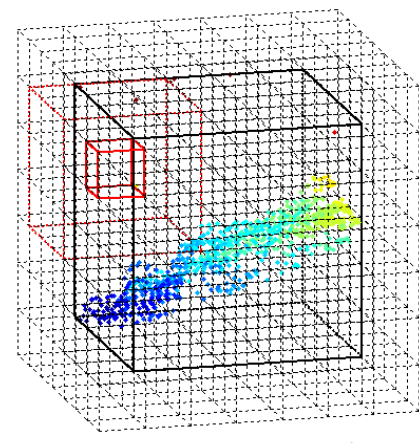

b. $V_{2}$ grid index and structure of window3D Schematic diagram

Figure 3. Schematic Diagram of 3D Grid and Window Structure

\subsection{Discrimination of Clustering Noise Points}

Because the Outlier clusters is the number of clusters isolated from the main body of the point cloud, by looking for each pieces of points in the cloud contains a seed grid for diffusion operations, and Count the number of points in each point cloud. According to the continuity of the laser Lidar point cloud, Keep the points with the largest number of point cloud is the main point cloud and remove the other point cloud could filtering out outlier clusters. Under the condition that the point data has been removed out which determined as the discrete noise point, recounting the number of points in each cubic grid in the cube grid attribute table M2, delete all the cubic grid which M2 (i, 4) $=0$ in table M2 after re statistics, set the cubic lattice of the first row in table M2 is a seed grid, that is $\mathrm{M} 2(1,5)=1$, traversing the M2 table in sequence from the first row in the table, the cube grid of M2(i, 5) >0 in the 26 cubic grids around the seed grid, together with the first operation of the seed grid as a seed grid for the second operation And so on, until the number of seed grids is no longer increased after $\mathrm{N}$ times operation. After diffuse the first 
point cloud, descending order the M2 table according to the value of last column M2(:,n+3), The value of 0 is that there is no diffusion operation of a little cube grid, placing these grids in the front of the table M2 again in order to diffusing operation, as the second sets of operation of the seed grid $M 2(1, n+3)=2$,repeat the first set of diffusing operation steps and so on, until all the value of the last column of the M2 table are not 0 . To statistics the number of cubic grid points involved according to the last column of the M2table, keep with the most points of point cloud clusters that filter out the outlier clusters. The diffusing algorithm schematic diagram in 2D as shown in Figure 4.

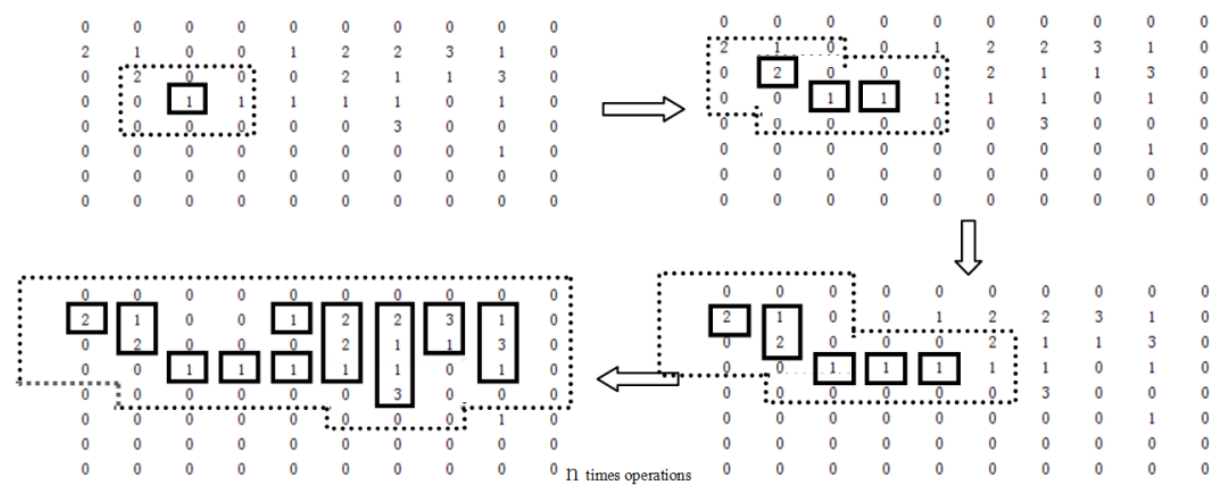

Figure 6. The Diffusing Algorithm Schematic Diagram in 2D

\section{Experimental Results and Analysis}

The average point cloud spacing reflects the overall distribution density of point cloud data points. Therefore, the average spacing based on Grid, according to the average distance between the grids can make the point cloud data of different density can be applicable to the unified division Rules.

In order to verify the effectiveness of the denoising algorithm, In this paper, the reference data of ISPRS is used as the experimental data. The experiments were conducted with 3 different sets of point cloud data, among them, the sample 1 and sample 2 are selected from the point cloud of the terrain covered with forest, Point cloud points number, density respectively:34496(1.785 points Per square meter)、25311(1.089 points Per square meter), Sample 3 is to select the terrain flat towns and villages point cloud data, Point cloud points number, density respectively: 179451(15.385points Per square meter), And each point cloud contains discrete noise with different number of isolated outliers and outlier clusters. The average spacing as the basis for the processing of 3D grid environment construction under different grid spacing coefficient, and the accuracy of the whole denoising effect is calculated by the accuracy of the discrete noise points. Results show that the discrete noise judgment when the length of cubic grid 0.11-0.13 times the average spacing when more accurate (As shown in Figure 5), the accuracy rate of noise discrimination is the ratio of the number of the actual number of the noise points and the number of the noise points. 


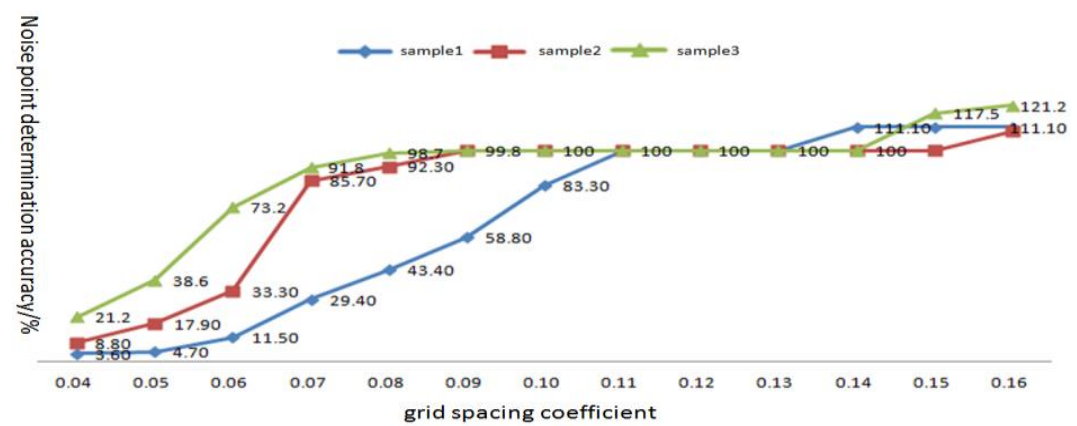

\section{Figure 5 Noise Discrimination Accuracy and Grid Spacing Coefficient Diagram}

Based on the above statistical results, the Experiment use 0.125 times average spacing as the standard 3D grid for operating, the results are shown in Figure 6:

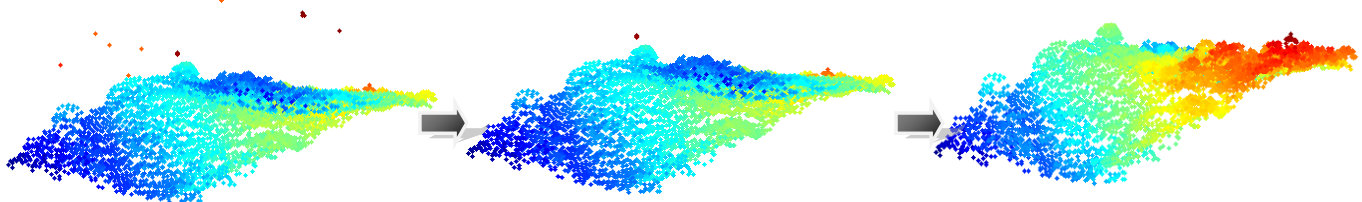

Woodland point cloud denoising (sample 1)

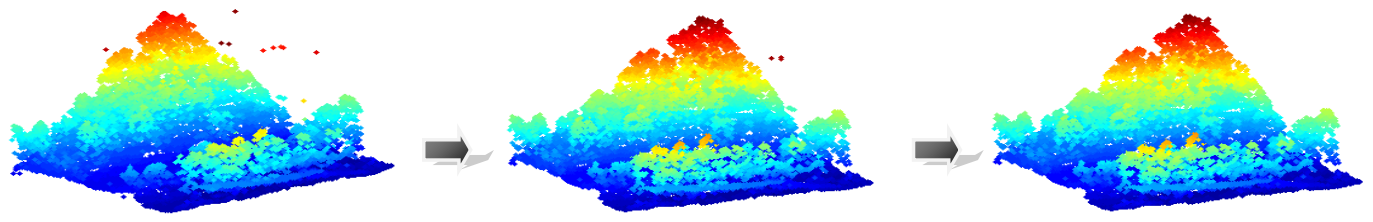

Woodland point cloud denoising (sample 2)

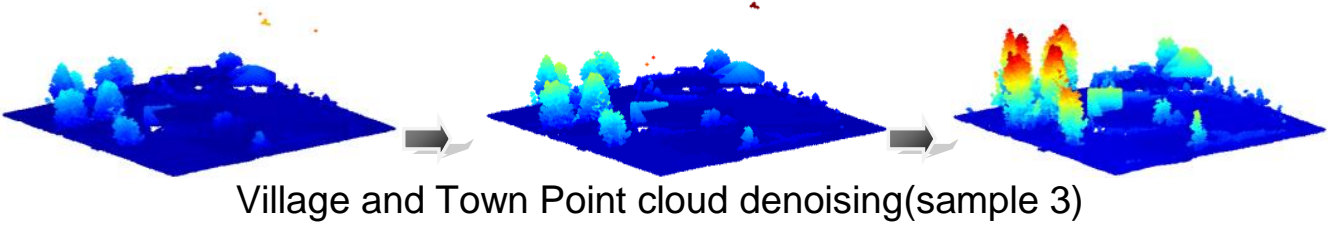

Figure 6. Point Cloud Denoising Effect Chart

\section{Conclusion}

This paper proposes a denoising algorithm for Airborne LIDAR point cloud based on $3 \mathrm{D}$ grid. The algorithm takes the average point cloud spacing for the construction of three-dimensional grid reference, to determine the discrete noise point and point cloud body according to the characteristics of spatial neighborhood between three-dimensional grid units, removing the isolated outliers and outlier clusters generated in terrain scanning.

The experments result show that this method is different from the traditional denoising algorithm, according to establishing 3D space grid for denoising. It provides a new way for Airborne LIDAR point cloud denoising.

\section{Acknowledgment}

This work was supported by the National Natural Science Foundation of China(Grant No.41472243). 


\section{References}

[1] Y. Livny, F. Yan and M. OLSON, "Automatic reconstruction of tree skeletal structures from point clouds", ACM Transactions on Graphics: Proceedings of ACM SIGGR APH Asia, vol. 29, no. 6, (2010).

[2] X.-M. Zhou and Q.-H. Ma, "Research and application of Airborne LIDAR point cloud data filtering algorithm “, The PLA Information Engineering University, (2011)

[3] Y. Zhang and L. Yan, "3DDiffusion filtering method of intensity noise for terrestrial laser scanning point cloud”, Acta Geodaetica et Cartographica Sinica, vol. 42, no. 4, (2013), pp. 568-573.

[4] L. Cheng and J.-Y. Gong, "Building Boundary Extraction Using Very High Resolution Images and LiDAR", Acta Geodaetica et Cartographica Sinica, vol. 3, (2008), pp. 391-393.

[5] J. T. Kwok, "I. Fast computation of 2-dimentional depth contours", Proc. KDD, (1998)

[6] J.-H. Nie, Y.-M. Hu and M. Ma, "Outlier Detection of Scattered Point Cloud by Classification", Journal of Computer-Aided Design \& Computer Graphics, vol. 9, (2011), pp. 1526-1532.

[7] S. Cao, J.-P. Yue and M. Wen, "Bilateral filtering denoise algorithm for point cloud based on feature selection", Journal of Southeast University(Natural Science, vol. 2, (2013), pp. 351-354.

[8] Q.-Y. Zhang, H. Chen and S. Zhu, "Application of density clustering algorithm in the continuous distribution point cloud denoising", Geospatial Information, vol. 6, (2011), pp. 101-104+2+1.

[9] J.-F. Zhu, X.-Y. Hu, Z.-X. Zhang and X.-D. Xiong, "Hierarchical outlier detection for point cloud data using a density analysis method", Acta Geodaetica et Cartographica Sinica, vol. 3, (2015), pp. 282-291.

\section{Author}

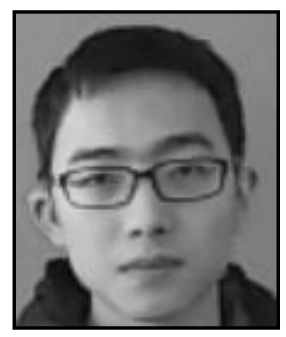

Shan Yong-hua(1992 ), he is MA.Sc.Candidate,Mainly engaged in GIS Applcation Research.

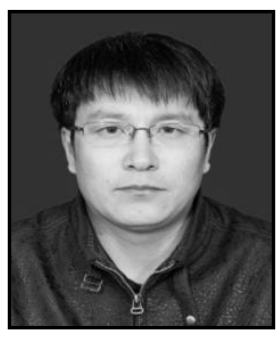

Zhang Xu-qing(1971 ), he is a PH.D of Jilin University,Mainly engaged in GIS Application Research.

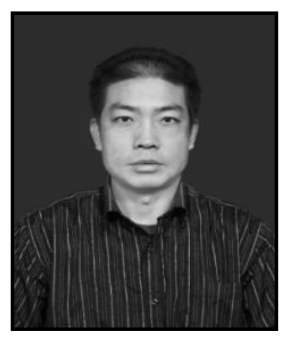

Niu Xue-feng(1970 ), he is a Professor of Jilin University,Mainly engaged in the research of photogrammetry and remote sensing.

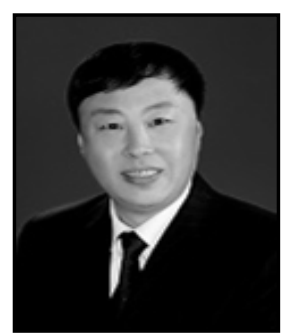

Yang Guo-dong(1963 ), he is a Professor of Jilin University,master instructor,mainly engaged in interdisciplinary research of surveying and mapping, geographic information systems, remote sensing, etc. 
International Journal of Signal Processing, Image Processing and Pattern Recognition Vol. 10, No. 3 (2017)

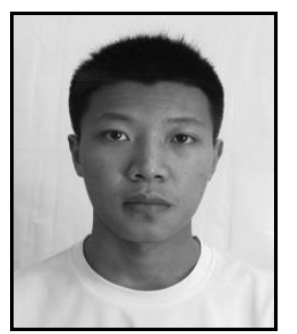

Zhang Ji-kai(1992 ), he is a MA.Sc.Candidate,Mainly engaged in GIS Applcation Research. 